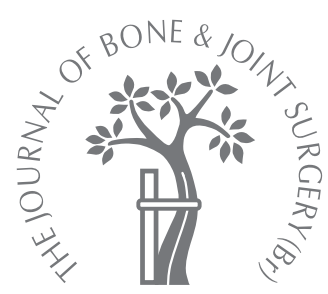

\title{
Visualisation of the pisotriquetral joint through standard portals for arthroscopy of the wrist
}

\author{
A CLINICAL AND ANATOMICAL STUDY
}
A. P. Arya,
R. Kulshreshtha,
G. K. Kakarala,
R. Singh,
J. P. Compson

From Kings College Hospital, London, England

\begin{abstract}
Disorders of the pisotriquetral joint are well recognised as the cause of pain on the ulnar side of the wrist. The joint is not usually examined during routine arthroscopy because it is assumed to have a separate joint cavity to the radiocarpal joint, although there is often a connection between the two.

We explored this connection during arthroscopy and in fresh-frozen cadaver wrists and found that in about half of the cases the pisotriquetral joint could be visualised through standard wrist portals. Four different types of connection were observed between the radiocarpal joint and the pisotriquetral joint. They ranged from a complete membrane separating the two, to no membrane at all, with various other types of connection in between.

We recommend that inspection of the pisotriquetral joint should be a part of the protocol for routine arthroscopy of the wrist.
\end{abstract}

Disorders of the pisiform bone and the pisotriquetral joint are well recognised as causes of pain on the ulnar side of the wrist. They include acute fractures of the pisiform, ${ }^{1}$ malunion and nonunion of the pisiform, pisotriquetral degenerative changes, ${ }^{2,3}$ loose bodies in the pisotriquetral joint, ${ }^{4}$ maltracking of the pisiform, pisotriquetral instability and the pisotriquetral pain syndrome after release of the carpal tunnel. ${ }^{5}$ Disorders of the pisotriquetral joint have been investigated by various methods including radiography, isotope bone scanning and MRI, but there have been no reports of the use of arthroscopy as an investigative tool for visualisation of this joint.

Arthroscopically, the wrist consists of four separate joints: the radiocarpal, midcarpal, distal radioulnar and the pisotriquetral joint. The radiocarpal and midcarpal joints are routinely visualised in arthroscopy of the wrist. The distal radioulnar joint can be seen either by using a smaller arthroscope from a separate portal or through the opening found in some types of triangular fibrocartilage tear. There is, however, little awareness of the existence of the pisotriquetral joint as a separate joint or that it often communicates with the radiocarpal joint. Textbooks ${ }^{6-8}$ on arthroscopy barely mention the pisotriquetral joint or the technique of its visualisation. Direct arthroscopy of the joint is difficult because of its small size and the risk of damage to the dorsal branch of the ulnar nerve. ${ }^{9}$ Visualisation of the pisotriquetral joint from the main radiocarpal joint would avoid this risk and potentially make the procedure easier.

We have examined the gross and arthroscopic anatomy of the connection between the radiocarpal joint and the pisotriquetral joint, its variability and the frequency of its visualisation during arthroscopy of the wrist.

\section{Patients and Methods}

We attempted to visualise the pisotriquetral joint in 98 patients who underwent wrist arthroscopy for various conditions between February 2003 and April 2006 (Table I). The type of connection between the radiocarpal joint and pisotriquetral joint was recorded.

There were 44 males and 54 females with a mean age of 36 years (12 to 68). Most were young adults (Table II). In 60 (62\%) the right wrist underwent arthroscopy.

Operative technique. All the arthroscopies were performed by the senior author (JPC) using the same method with the wrist suspended by finger traps and with $2.27 \mathrm{~kg}$ (5 lbs) of countertraction. A $2.7 \mathrm{~mm}$ arthroscope was introduced through the $6 \mathrm{R}$ portal $^{9}$ to visualise the pisotriquetral joint. ${ }^{9}$ The triangular fibrocartilage was used for orientation and the lunate was identified. The arthroscope was swept dis- 
Table I. Indications for wrist arthroscopy in the 98 patients

\begin{tabular}{lcl}
\hline & Number (\%) \\
\hline Pain in the ulnar side of the wrist & $45(46)$ \\
Dorsal wrist pain & $27(28)$ \\
Lunate-related disorders & $7(7)$ \\
Scaphoid-related disorders & $9(9)$ \\
Miscellaneous & $10(10)$ \\
Total & $98(100)$ \\
\hline
\end{tabular}

Table II. Distribution of patients by age and gender

\begin{tabular}{lccc}
\hline Age (yrs) & Number & Male & Female \\
\hline$<20$ & 9 & 2 & 7 \\
20 to 40 & 58 & 29 & 29 \\
$>40$ & 31 & 13 & 18 \\
Total & 98 & 44 & 54 \\
\hline
\end{tabular}

Table III. Incidences of the different types of pisotriquetral joint

\begin{tabular}{lll}
\hline Type of joint & \multicolumn{2}{c}{ Number (\%) } \\
\hline I & 23 & $(27)$ \\
II & 15 & $(17)$ \\
III & 7 & $(8)$ \\
IV & 42 & $(48)$ \\
Total & $87^{*}$ & $(100)$ \\
\hline
\end{tabular}

*in 11 patients, the pisotriquetral joint could not be accessed lated and the types of connection between the radiocarpal joint and pisotriquetral joint were noted.

\section{Results}

We were able to look for pisotriquetral joint in 87 of the 98 wrists. In 11 the expected site of the pisotriquetral joint could not be accessed either because of post-traumatic or postsurgical fibrosis, or synovitis. In the remaining 87 , one of the following four types of connection between the radiocarpal and pisotriquetral joint was found (Table III; Fig. 1).

Type I (23 patients, 27\%). A thick synovial membrane covered the joint which made it impossible to visualise (Fig. 1a).

Type II (15 patients, 17\%). A synovial membrane similar to that of type I covered the joint, but was thin enough to see the silhouette of the pisiform bone but not the joint itself (Fig. 1b).

Type III (7 patients, $8 \%$ ). The synovial membrane covering the joint was present, but was fenestrated. There was a small opening, but it was not possible to pass the arthroscope through it (Fig. 1c).

Type IV (42 patients, 48\%). Either no synovial membrane was present or it had a large fenestration through which the arthroscope could easily enter the pisotriquetral joint which was clearly visualised (Fig. 1d).

Similar findings were found on dissection of the cadaver wrists. It was possible to pass the probe from the region of the $6 \mathrm{R}$ portal to the pisotriquetral joint. Gross examination and observation of a cross-section of the pisotriquetral joint revealed a well-formed circumferential synovial membrane (Fig. 2) in four cadavers, with a horseshoe-shaped and incomplete synovial membrane in the remaining four.

\section{Discussion}

The pisotriquetral joint is the smallest of the four joints of the wrist. Although separate, it is often connected to the radiocarpal joint through a fenestration. The gross anatomy and kinematics of the pisotriquetral joint have been well described. ${ }^{1,2,10-12}$ It is a synovial diarthrosis and is enclosed in a small, but usually loose and tough capsule. For many years, the pisiform bone was considered to be a vestigial structure, but recent kinematic studies have indicated that its function is probably similar to that of the patella, in that it extends the lever arm of the tendon of flexor carpi ulnaris away from the centre of the rotation of the wrist and increases the flexor force. ${ }^{4}$

Filling of the pisotriquetral joint during arthrography of the proximal wrist is a common finding which occurs in approximately one-third of normal wrists. ${ }^{13}$ The connection between the proximal wrist and the pisotriquetral joint has also been noted in anatomical studies. ${ }^{14,15}$ Viegas et $\mathrm{al}^{12}$ found such a connection in $88 \%$ of 76 fresh cadaver wrists, but they were not certain whether it represented a normal anatomical variation or a pathological finding.

The pisotriquetral joint is well recognised as a site of pain on the ulnar side of the wrist. ${ }^{1-5}$ Yamaguchi et al ${ }^{2}$ described 


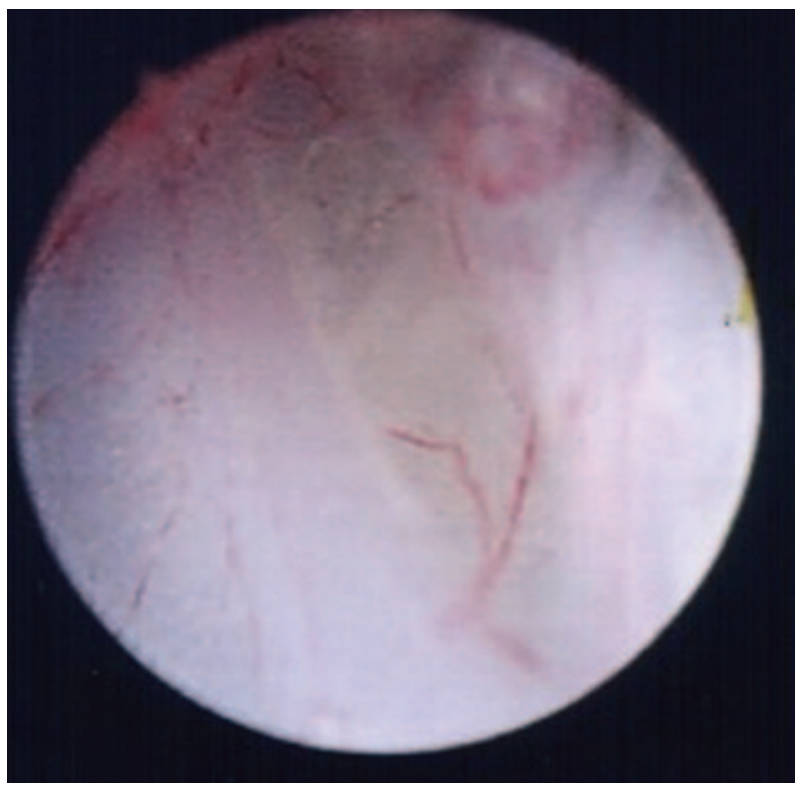

Fig. 1a

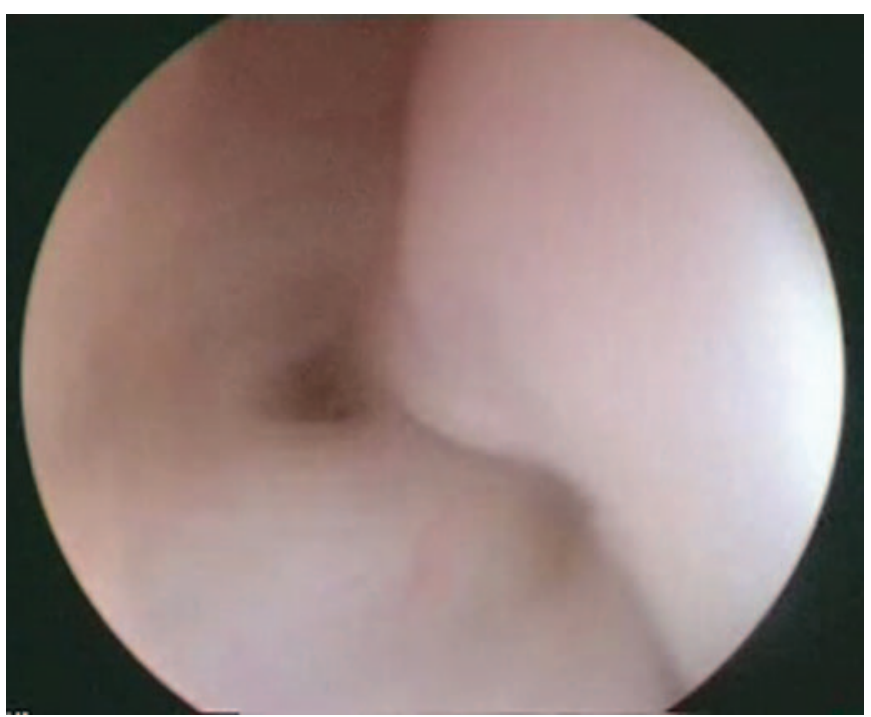

Fig. 1c

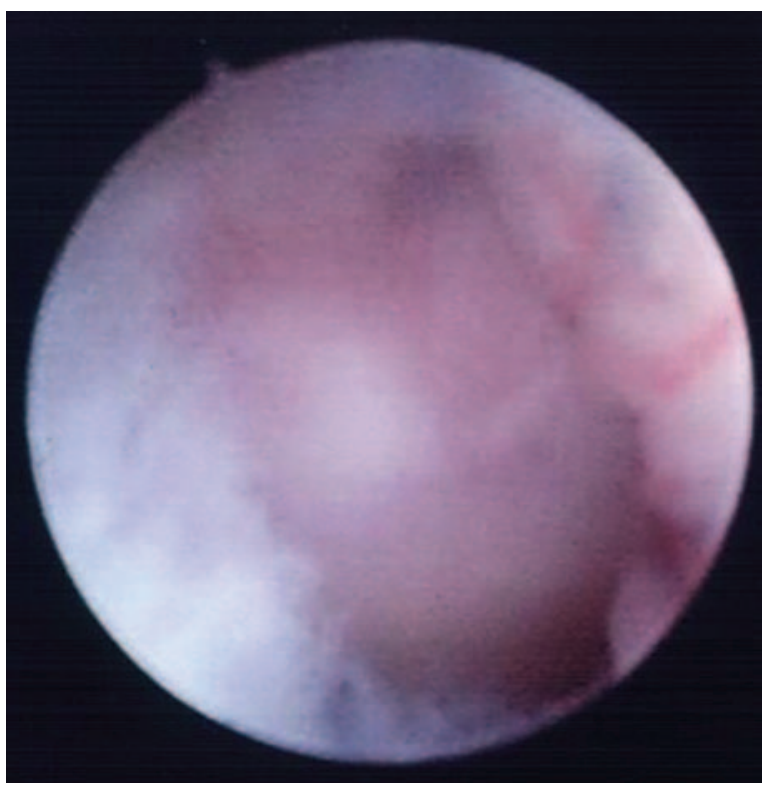

Fig. 1b

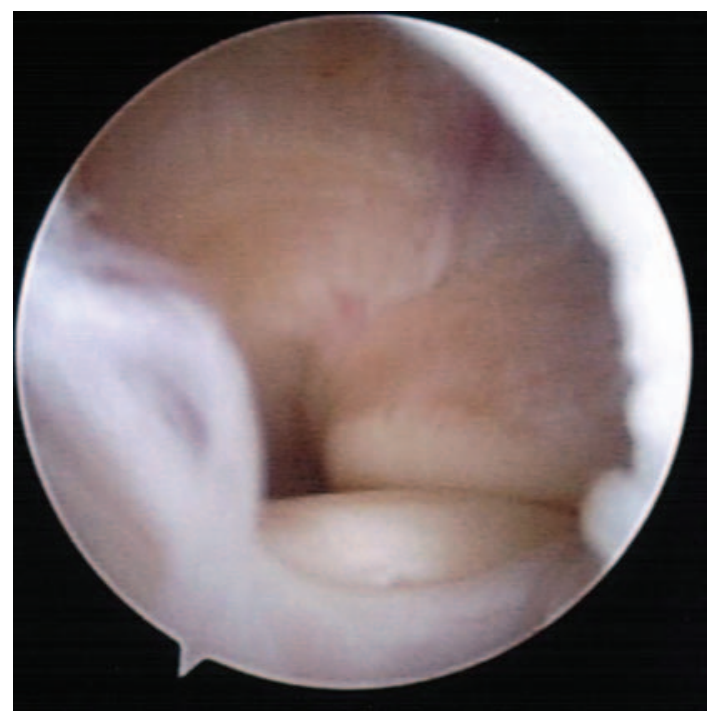

Fig. 1d

Arthroscopy showing a) type I, b) type II, c) type III and d) type IV connection between the pisotriquetral and the radiocarpal joint.

various patterns of degenerative change in the pisotriquetral joint in a study of 80 cadaver wrists. Arthroscopy is a reliable means of diagnosing intra-articular conditions in the wrist, particularly wear or damage to the articular surface. However, there have been few reports on arthroscopy of the pisotriquetral joint. Whipple et $\mathrm{al}^{7}$ described the appearance of the normal pisotriquetral joint seen on arthroscopy through an aperture in the ulnar capsule, located slightly volar and distal to the prestyloid recess.
They commented that, when present, this represented a normal variant which afforded the examiner an opportunity to look for synovial hypertrophy or marginal articular degeneration in the pisotriquetral joint. In another report, Whipple, Poehling and Roth ${ }^{16}$ postulated that visualisation of the pisiform bone is occasionally possible in the normal wrist and, if it is very evident, a tear of the ulnotriquetral ligament must be considered. We disagree, and believe that 


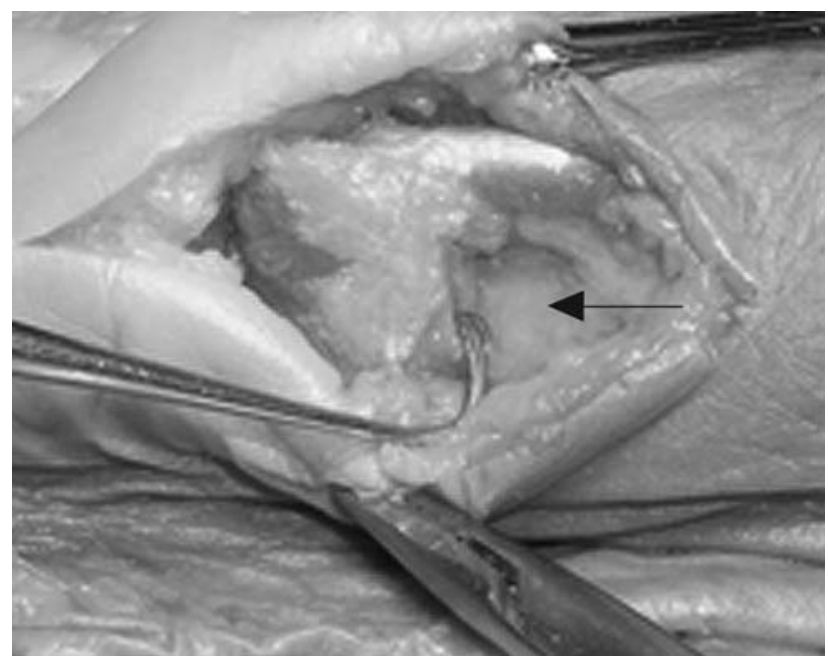

Fig. 2

Photograph showing a thin synovial membrane covering the pisotriquetral joint.

this underestimates the information which may be potentially gained, since we have been able to see most of the joint and especially the surface of the pisiform bone in about half of our cases. We do not consider that this visualisation of the pisotriquetral joint is the result of trauma, but rather is due to the type of anatomical variant present. Arthroscopy of the wrist is gradually increasing in popularity and inspection of the pisotriquetral joint should be a routine part of the procedure particularly in the presence of pain on the ulnar side.

No benefits in any form have been received or will be received from a commercial party related directly or indirectly to the subject of this article.

\section{References}

1. Vasilas A, Grieco RV, Bartone NF. Roentgen aspects of injuries to the pisiform bone and pisotriquetral joint. J Bone Joint Surg [Am]1960;42-A:1317-28.

2. Yamaguchi S, Viegas SF, Patterson RM. Anatomic study of the pisotriquetral joint: ligament anatomy and cartilaginous change. J Hand Surg [Am] 1998;23:600-6.

3. Miller MD. Review of orthopaedics. Fourth ed. Philadelphia: Saunders, 2004:395.

4. Hall TD. Loose body in the pisotriquetral joint. J Bone Joint Surg [Am] 1981;63A:498-500.

5. Seradge H, Seradge E. Piso-triquetral pain syndrome after carpal tunnel release. J Hand Surg [Am] 1989;14:858-62.

6. Stanley J, Saffar P. Wrist arthroscopy. London: Martin Dunitz Ltd., 1994:79-91.

7. Whipple TL, Cooney WP III, Osterman AL, Viegas SF. Wrist arthroscopy. Instr Course Lect 1995;44:139-45.

8. Solomon L, Warwick DJ, Nayagam S. Apley's system of orthopaedics and fractures. Eighth ed. London: Arnold, 2001:316-19.

9. Tindall A, Patel M, Frost A, et al. The anatomy of the dorsal cutaneous branch of the ulnar nerve: a safe zone for positioning of $6 \mathrm{R}$ portal in wrist arthroscopy. J Hand Surg [Br] 2006;31:203-5.

10. Taleisnik J. The wrist. New York: Churchill Livingstone, 1985:1-12.

11. Pevny T, Rayan GM, Egle D. Ligamentous and tendinous support of the pisiform, anatomic and biomechanical study. J Hand Surg [Am] 1995;20-A:299-304.

12. Viegas SF, Patterson RM, Hokanson JA, Davis J. Wrist anatomy: incidence, distribution and correlation of anatomic variations, tear and arthrosis. J Hand Surg [Am] 1993;18:463-75.

13. Bogumill GP. Anatomy of wrist. In: Lichtman DM, ed. The wrist and its disorders. Philadelphia: W B Saunders, 1988:21.

14. Lewis $\mathbf{O J}$, Hamshere RJ, Bucknill TM. The anatomy of the wrist joint. J Anat 1970;106:539-52.

15. Palmer AK, Werner FW. The triangular fibrocartilage complex of the wrist: anatomy and function. J Hand Surg [Am] 1981;6:153-62.

16. Whipple TL, Poehling GG, Roth JH. Surgical technique for wrist arthroscopy. In: Poehling GG, ed. Arthroscopy of the wrist and elbow. New York: Raven Press Ltd., 1994:63-71. 